\title{
Effect of Roll Fluting Disposition and Roll Gap on Breakage of Wheat Kernels During First-Break Roller Milling
}

\author{
Chaoying Fang ${ }^{1}$ and Grant M. Campbell ${ }^{1,2}$
}

\begin{abstract}
Breakage of wheat kernels during first-break roller milling depends on many factors, including the disposition of the fluted rolls: sharp-to-sharp, sharp-to-dull, dull-to-sharp, or dull-to-dull. Wheat kernel breakage under different dispositions during milling was studied using high-speed video imaging. The results show significant slippage between kernels and the flutes when a dull working angle is used, especially when the dull angle is on the fast roll. Experiments were conducted to compare the size distributions resulting from the four dispositions. Representative hard and soft wheat cultivars were milled using fluted rolls at five different roll gaps, and the resulting size distribution of the milled stocks was
\end{abstract}

ABSTRACT measured by sieve analysis. A sharp-to-sharp disposition gave a relatively uniform or straight line size distribution over the particle size range of $212-2,000 \mu \mathrm{m}$. By contrast, a dull-to-dull disposition gave a Ushaped distribution with more larger and smaller particles and fewer in the middle size range. The size distributions from the other two dispositions lay between these, giving a transition from a straight-line distribution for sharp-to-sharp disposition, slightly U-shaped for sharpto-dull, more curved for dull-to-sharp, and highly U-shaped for dull-todull. The effect of roll gap was to change the balance between large and small particles.
The particle size distribution resulting from first-break roller milling of wheat is of critical importance because it directly affects the mill flows and effectiveness of the rest of the milling process. A review of the literature by Fang and Campbell (2002) indicated that much research has been performed on various factors affecting first-break roller milling of wheat, including physicochemical properties of the wheat kernels and aspects of roll design and operation. However, relatively little work has addressed the effect of roll disposition on the breakage patterns of wheat kernels and the resulting particle size distribution delivered by first-break roller milling.

In an accompanying article (Fang and Campbell 2002), we applied stress-strain analysis to identify the fracture mechanics principles describing wheat kernel breakage during milling with fluted rolls. By studying the deformation of a wheat kernel engaged between two fluted rolls in sharp-to-sharp (S-S) disposition, the planes of principal stresses and maximum shear stresses along which the kernel is most likely to be broken were calculated. As tensile strength is much smaller than compressive strength (Glenn et al 1991; Glenn and Johnston 1992), the most probable fracture plane was calculated to be that perpendicular to the principal tensile stress. The effects of roll gap, differential, and roll diameter on the stresses and strains were also discussed. High-speed video pictures of wheat kernel breakage under sharp-to-sharp milling were presented to illustrate the breakage patterns and fracture planes occurring during kernel breakage.

The objective of this study was to investigate the effect of roll disposition on the particle size distribution of wheat after first-break roller milling. The equations developed previously (Fang and Campbell 2002) to describe breakage under sharp-to-sharp milling are applied to other roll dispositions: sharp-to-dull (S-D), dull-to-sharp (D-S), and dull-to-dull (D-D). High-speed video pictures of wheat breakage are presented to illustrate the fracture events occurring at different roll dispositions. Experimental measurements of the size distributions arising from first-break roller milling of two representative hard and soft wheat cultivars under different dispositions and roll gaps are also presented and discussed in terms of the breakage patterns suggested by fracture mechanics and observed by high speed video.

\footnotetext{
${ }^{1}$ Satake Centre for Grain Process Engineering, Department of Chemical Engineering, UMIST, Manchester, M60 1QD, UK.

${ }^{2}$ Corresponding author. Phone: +44 (0)161 2004472 . Fax +44 (0)161 2004399. E-mail: g.campbell@umist.ac.uk.
}

Publication no. C-2002-0604-03R.

(C) 2002 American Association of Cereal Chemists, Inc.

\section{STRESSES AND STRAINS ON A KERNEL UNDER DIFFERENT ROLL DISPOSITIONS}

In an accompanying article (Fang and Campbell 2002), the planes of principal compressive and tensile strain and maximum shear strain were calculated for a kernel, assumed for simplicity to be a spherical particle, under a sharp-to-sharp disposition. Based on the calculated compressive and shear strains, the principal strains $\varepsilon_{1}$ and $\varepsilon_{2}$, the maximum shear strain $\gamma_{\max }$ and the planes along which they occur can be calculated by

$$
\begin{gathered}
\varepsilon_{1,2}=-\frac{\varepsilon_{x}}{2} \mp \sqrt{\left(\frac{-\varepsilon_{x}}{2}\right)^{2}+\left(\frac{\gamma_{x y}}{2}\right)^{2}} \\
\frac{\gamma_{\max }}{2}=\sqrt{\left(\frac{-\varepsilon_{x}}{2}\right)^{2}+\left(\frac{\gamma_{x y}}{2}\right)^{2}}
\end{gathered}
$$

The relevant principal stresses and the maximum shear stresses can also be calculated. Equation 1 shows that $\varepsilon_{2}>0$ is a tensile strain that arises because of the existence of the shear strain. The kernel was most likely to fracture along the plane of maximum tensile stress because of the weak tensile strength of the endosperm. The orientations of the planes of principal stress and maximum shear stress were calculated by

$$
\begin{gathered}
\theta_{p 1}=\frac{1}{2} \tan ^{-1}\left(\frac{\gamma_{x y}}{-\varepsilon_{x}}\right) \\
\theta_{p 2}=\theta_{p 1}+90^{\circ} \\
\theta_{S \& S^{\prime}}=\theta_{p 1} \pm 45^{\circ}
\end{gathered}
$$

The origin of the tensile stress is the shear forces exerted on the kernel by the two rolls due to their differential speeds. Under sharpto-sharp milling, no slippage occurs between the fast roll flute and the kernel before breakage. When the two rolls are in other dispositions, the shear forces and shear stresses acting on the kernel are different, and slippage may occur. Figure 1 shows the forces acting on the kernel by two fluted rolls in the four different dispositions. When the dull face of the flute is the working edge, the shear force on a kernel relies largely on the friction between the kernel and the flute surface. It will thus be much smaller than when 


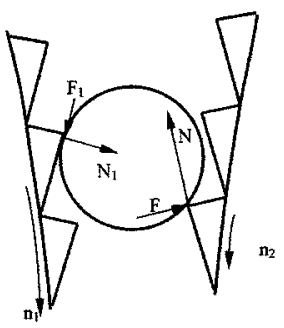

S-S

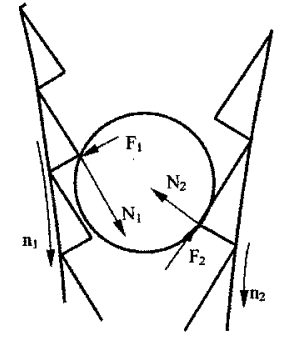

S-D

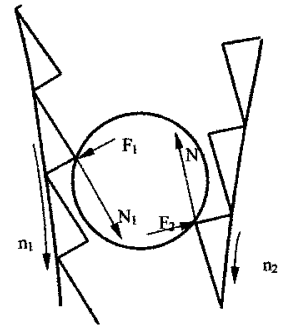

D-S

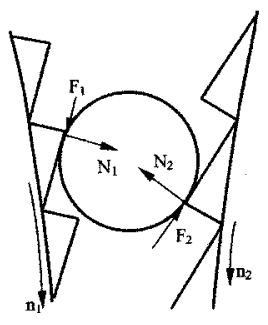

D-D

Fig. 1. Forces acting on a spherical particle engaged between two rolls with different roll dispositions: sharp-to-sharp (S-S); sharp-to-dull (S-D); dull-tosharp (D-S); and dull-to-dull (D-D).

$0 \mathrm{~ms}$

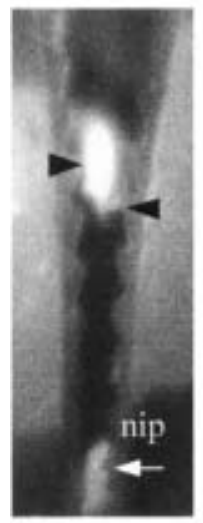

A
$4.5 \mathrm{~ms}$

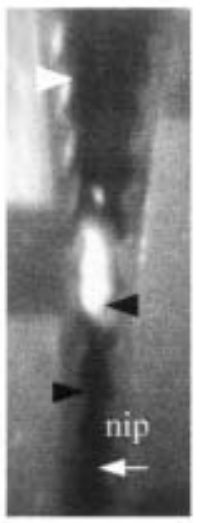

B
$5.5 \mathrm{~ms}$

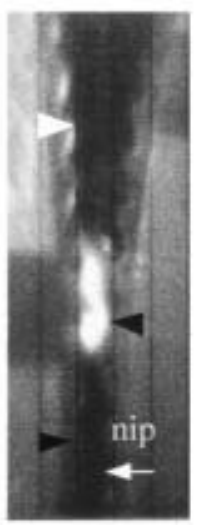

C

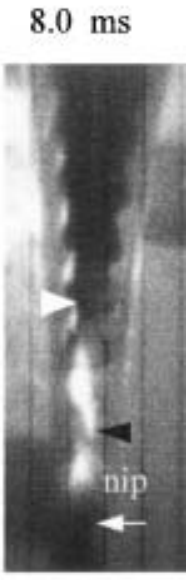

D

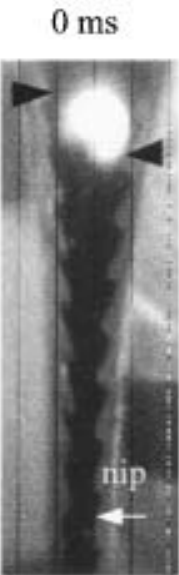

A

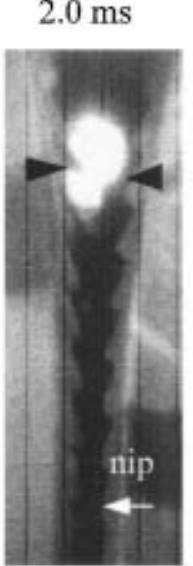

B

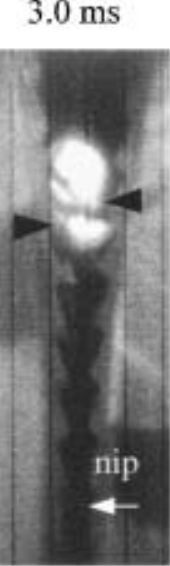

C
$6.0 \mathrm{~ms}$

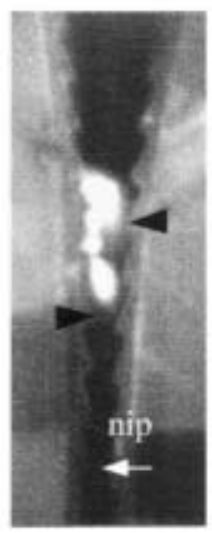

D

Fig. 2. An example of kernel breakage under dull-to-sharp (D-S) disposition.

the kernel is struck by the sharp working angle. If friction is insufficient to prevent slippage between the kernel and the flute, the shear strain of the kernel will be smaller than under the no-slippage condition obtained when the sharp face is the working edge.

\section{HIGH-SPEED VIDEO OBSERVATION AT DIFFERENT ROLL DISPOSITIONS}

Further observations of the breakage of wheat kernels under different roll dispositions were made as described previously (Fang and Campbell 2002). Figure 2 illustrates the process of a kernel being broken under a dull-to-sharp (D-S) disposition. The black arrows show the points of initial contact on each roll, while the white arrows on the fast roll are shown for reference.

Comparing the breakage shown in Fig. 2 with that shown for S-S in Fig. 10 of Fang and Campbell (2002) leads to four conclusions. 1) The breakage under D-S occurs $5.5 \mathrm{msec}$ after the kernel is initially engaged between the two rolls, which is much later than under $\mathrm{S}-\mathrm{S}(0.5 \mathrm{msec}) .2)$ The kernel is significantly compressed before a clear fracture event is evident. 3) There is slippage between the kernel and the flutes of the fast roll. Comparing Fig. 2A and C, if no slippage occurred, the leading end of the kernel should be in the position of the initial contacting point on the fast roll, indicated by the black arrow. However, after $5.5 \mathrm{msec}$, it has only just overtaken the initial contacting point on the slow roll. The extent of slippage is $>80 \%$. This means the shear strain experienced by the kernel is $<20 \%$ of that in S-S. 4) There is not a succession of discrete breakage events as occurred under S-S; instead the kernel and subsequent fragments are primarily crushed rather than divided.

Equation 1 shows that with shear strain reducing, the principal tensile strain $\varepsilon_{2}$ decreases. If shear strain is zero, the $\varepsilon_{2}$ will be zero

Fig. 3. Kernel breakage under sharp-to-dull (S-D) disposition.

too. With smaller $\varepsilon_{2}$, the kernel may not break because of tensile stress. Instead, it will break by compressive stress. The orientations of the planes with principal stress and maximum shear stress can be calculated by Equations 3-5. If using the same example as in Fang and Campbell (2002), when $\alpha_{1}=9^{\circ}, D=4 \mathrm{~mm}, R=125$ $\mathrm{mm}, G=0.5 \mathrm{~mm}, \Delta n=2.7$, and $d \alpha_{1}=\alpha_{1}-\alpha_{2}=0.25^{\circ}$, but with $20 \%$ of the shear strain, the $\theta_{p 1}$ will change from $32^{\circ}$ to $11^{\circ}$ and $\theta_{p_{2}}$ from $58^{\circ}$ to $79^{\circ}$. This means that if a kernel breaks by tensile stress, the breakage plane will be closer to the $x$-axis direction, but if the kernel breaks by compressive stress, the breakage plane will be closer to the $y$-axis direction. Figure 2 shows that the kernel is actually crushed by compressive stress but the fracture plane could not be seen until the kernel approaches the roll nip.

Figures 3 and 4 show the breakage of kernels under S-D and D$\mathrm{D}$ dispositions. The breakage patterns are similar to the cases of S$\mathrm{S}$ and D-S, respectively. However, because the working edge of the flutes on the slow roll is at a dull angle, slippage between kernel and flute occurs. Figure 4 illustrates clearly that the kernel slipped relative to both the fast and slow rolls. This slippage inevitably reduces the shear strain applied to the kernel.

Considering the above examples and numerous others observed by high-speed video, it appears that during grinding of wheat using fluted rolls, two types of breakage occur. The first type involves a clean breakage of the kernel or its progeny particles into two smaller fragments. This type of fracture breaks both bran and endosperm together, and opens up the kernel to expose the endosperm, but the endosperm does not shatter to a great extent; it can be described as kernel-opening breakage, and occurs when shear and tensile forces are dominant. The second type of breakage involves primarily crushing forces. Under compression, the bran carries little load and serves primarily to contain the structure within the grain (Arnold and 
Roberts 1966). These crushing forces therefore shatter the brittle endosperm into many small fragments but leave the tougher bran more intact, with endosperm material remaining adhering to the bran particles. This type of breakage can be referred to as endospermrelease breakage, and occurs when compressive forces dominate. These compressive forces arise either due to the relative angles of the flutes (they are greatest for D-D disposition) or when a particle approaches the nip.

The balance between kernel-opening breakage and endospermrelease breakage determines the size distribution of the milled wheat particles. For first-break roller milling, the grinding zone is from the point where a kernel is initially engaged between two rolls to the roll nip (the point at which the gap is at a minimum). For a pair of rolls with a diameter of $250 \mathrm{~mm}$ and a roll gap of $0.5 \mathrm{~mm}$, a kernel with a thickness of $4 \mathrm{~mm}$ would result in a grinding zone of $\approx 21 \mathrm{~mm}$ in length. Breakage within this grinding zone can be divided into the kernel-opening period and the endosperm-release period. During the kernel-opening period, the kernel is deformed by shear such that

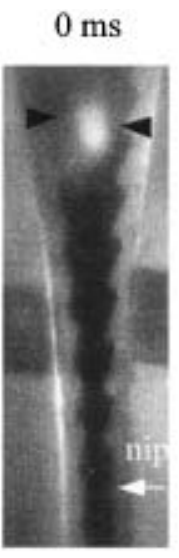

A

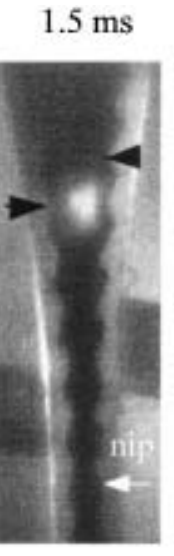

B

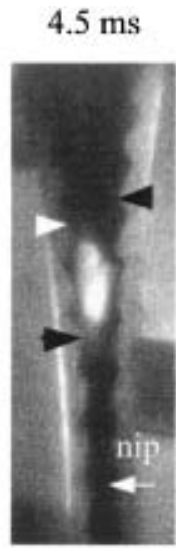

C

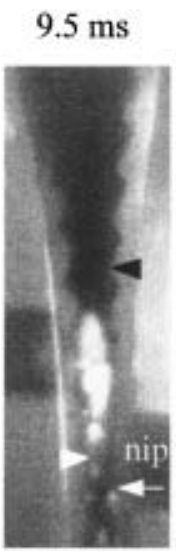

D

Fig. 4. Kernel breakage under dull-to-dull (D-D) disposition.
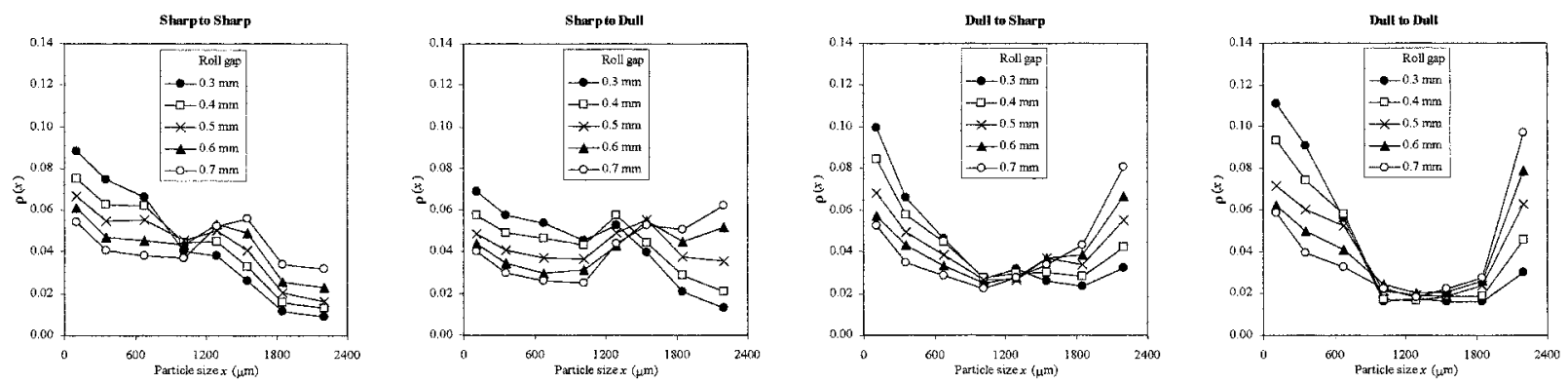

Hereward
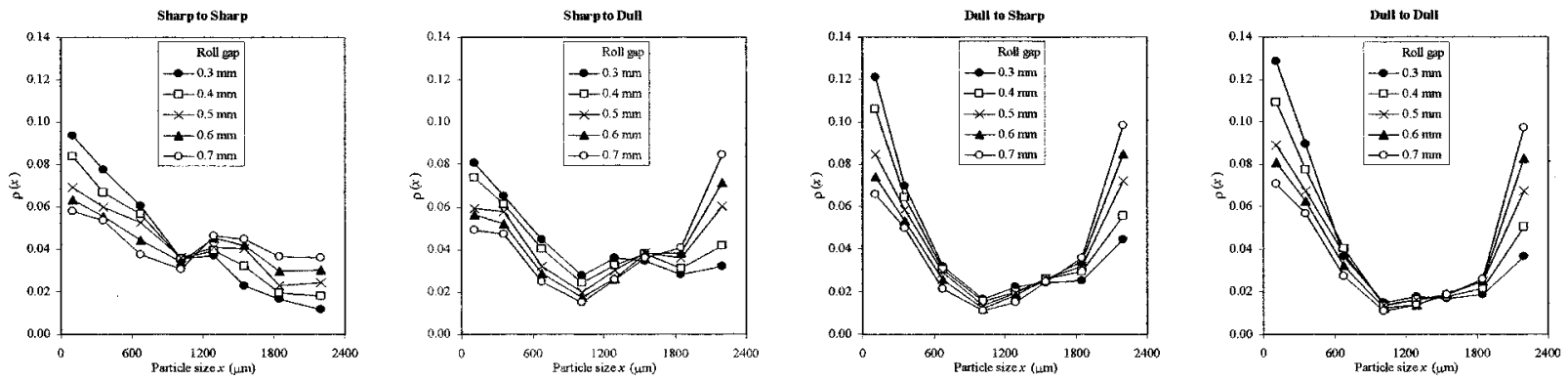

Consort

Fig. 5. Particle size distributions resulting from milling samples of Hereward (hard) and Consort (soft) wheats at different roll gaps under sharp-to-sharp (S-S); sharp-to-dull (S-D); dull-to-sharp (D-S); and dull-to-dull (D-D) disposition. 
milled in triplicate on the test roller mill (Satake STR-100) (Bunn et al 2001; Campbell et al 2001; Fang and Campbell 2002) for each cultivar at each of the four roll dispositions and at five roll gaps $(0.3,0.4,0.5,0.6$, and $0.7 \mathrm{~mm})$, giving a total of 120 samples. The roll gaps were set using a feeler gauge. The fast and slow roll speeds were 600 and $222 \mathrm{rpm}$, respectively, giving a differential of 2.7. Fluted rolls $100 \mathrm{~mm}$ in length with 10.5 flutes per inch were used, with flute angles of $\beta_{\mathrm{s}}=21^{\circ}$ and $\beta_{\mathrm{d}}=67^{\circ}$. The working angle combinations for the four roll dispositions are listed in Table I. The mill was operated at a feed rate of $\approx 375-500 \mathrm{~kg} / \mathrm{hr}$, corresponding to $3,750-5,000 \mathrm{~kg} / \mathrm{m} / \mathrm{hr}$ on a full-length commercial mill. The entire milled stocks from each trial were collected for sieve analysis. Samples were sieved for $8 \mathrm{~min}$ on a Simon sifter operating at 190 rpm with a throw of $7.5 \mathrm{~cm}$, using 200-mm diameter wire mesh sieves of 2,000, 1,700, 1,400, 1,180, 850, 500, and $212 \mu \mathrm{m}$, along with a bottom pan.

The probability density function, $\rho(x)$ (units: $\mu \mathrm{m}^{-1}$ ), describing the mass-based particle size distribution was represented by the mass fraction of particles staying on each sieve in the stack divided by the difference between the aperture size of this sieve and that of the adjacent larger sieve. For the fraction $>2,000 \mu \mathrm{m}$, the proportion was divided by the difference between 2,000 and 2,400 $\mu \mathrm{m}$, based on experience that few particles $>2,400 \mu \mathrm{m}$ are produced.

\section{RESULTS AND DISCUSSION}

Figure 5 shows the particle size distributions resulting from milling samples of Hereward (hard) and Consort (soft) wheat at different roll gaps under S-S, S-D, D-S, and D-D dispositions.

For S-S disposition, kernels undergo primarily kernel-opening breakage, with fracture occurring not only of endosperm but also of the bran. The resulting size distributions shown in Fig. 5 can be described approximately over a wide range by straight lines of negative slope. The proportion of particles in a given size range is indicated by the area under the curves. For both Hereward and Consort, first-break roller milling results in a greater proportion (by mass) of small particles than of large particles. As roll gap increased from 0.3 to $0.7 \mathrm{~mm}$, less breakage occurred, resulting in more large particles and fewer small particles, and thus changing the slope of the line.

The D-D disposition allows slippage between kernel and flutes and therefore imparts less shear stress and proportionally greater compression. Endosperm-release breakage thus dominates, resulting in substantial shattering and fragmentation of the endosperm but leaving the bran layers with adhering endosperm flattened and largely intact. Thus, the particle size distributions shown in Fig. 5 for D-D milling of both wheats are highly U-shaped with many small particles (shattered endosperm) and many large particles (intact bran particles), and relatively little material in the middle size ranges. Again, the effect of roll gap is to change the balance between small and large particles. The delivery of a U-shaped distribution with distinct populations of large and small particles facilitates separation of branny and endosperm particles during subsequent plansifting. This is undoubtedly the reason that a dull-to-dull first-break disposition is preferred by millers.

The particle size distributions resulting from S-D and D-S milling lay between the extremes given by S-S and D-D, showing

TABLE I

Working Angles for Different Roll Dispositions

\begin{tabular}{lcc}
\hline & \multicolumn{2}{c}{ Working Angle } \\
\cline { 2 - 3 } Roll Disposition & Fast Roll & Slow Roll \\
\hline Sharp-to-Sharp (S-S) & $21^{\circ}$ & $21^{\circ}$ \\
Sharp-to-Dull (S-D) & $21^{\circ}$ & $67^{\circ}$ \\
Dull-to-Sharp (D-S) & $67^{\circ}$ & $21^{\circ}$ \\
Dull-to-Dull (D-D) & $67^{\circ}$ & $67^{\circ}$ \\
\hline
\end{tabular}

a transition from kernel-opening-dominated to endosperm-releasedominated breakage. The transition occurred more rapidly for the soft wheat, Consort, which also shows a greater proportion of smaller particles for a given roll gap and disposition, compared with the hard wheat. Soft wheat endosperm has weaker links between starch granules and the protein matrix, and disintegrates into small particles more readily (Haddad et al 1999). The outer bran layers lose their support and collapse without substantial breakage. Thus, soft wheat fracture is dominated by endosperm-release breakage patterns, resulting in a large break release from first-break roller milling and many large flattened bran particles.

Creason (1975) noted that "the grinding rolls are the heart of the milling operation", but lamented that little had been written or discussed on the subject of roll operation and the effects of corrugations. With respect to roll disposition, most work has been qualitative and descriptive. Our experimental results quantify the effects of roll disposition in terms of particle size distribution, and are consistent with the simple theoretical fracture mechanics analysis presented and with video observations. Breakage of wheat kernels during first-break roller milling is extremely complex. Nevertheless, fracture mechanics can be applied to interpret milling results and refine the milling operation through a better understanding of the effects of flute profiles and roll operation on the size distribution of particles produced at first-break roller milling.

\section{CONCLUSIONS}

Breakage of wheat kernels during first-break roller milling depends on the directions and magnitudes of the compressive, tensile and shear forces imparted due to the angles of the flutes and the differential speeds of the rolls. High-speed video imaging of wheat kernel breakage demonstrates no slippage between kernels and flutes before breakage for S-S milling but significant slippage relative to the dull working angle under other dispositions. Two types of breakage pattern can be identified: kernel-opening and endosperm-release. In kernel-opening breakage, both bran and endosperm are broken together to give few, large particles; successive breakage events result in a wide and relatively even range of particle sizes. In endospermrelease breakage, compressive forces cause disintegration of the fragile endosperm while leaving the bran relatively intact. S-S milling is dominated by kernel-opening breakage, resulting in a broad and relatively even distribution of particles, while endosperm-release breakage dominates during D-D milling, resulting in a U-shaped distribution with many small and large particles but few in the midsize range. S-D and D-S milling lie between these extremes, giving a transition from straight-line distributions for S-S, slightly curved for S-D, more curved for D-S, and highly U-shaped for D-D. Endosperm-release fracture predominates for softer wheats, resulting in a faster transition and a more distinctly U-shaped distribution. The effect of roll gap is to change the balance between large and small particles; as roll gap is increased, less breakage occurs, resulting in a greater mass fraction of large particles.

\section{ACKNOWLEDGMENTS}

This work was funded under EPSRC grant GR/M49939. We are also grateful for the loan of the high-speed video system from the EPSRC Equipment Pool Loan Scheme. The Satake Corporation of Japan is gratefully acknowledged for its support of these studies.

\section{LITERATURE CITED}

Arnold, P. C., and Roberts, A. W. 1966. Stress distributions in loaded wheat grains. J. Agric. Eng. Res. 11:38-43.

Bunn, P. J., Campbell, G. M., Fang, C.-Y., and Hook, S. C. W. 2001. On predicting roller milling performance. III. The particle size distribution from roller milling of various wheats using fluted rolls. Proc. 6th World Congress of Chemical Engineering. University of Melbourne: Melbourne, Australia. 
Campbell, G. M., Fang, C. Y, Bunn, P. J., Gibson, A. A., Thompson, F., and Haigh, A. 2001. Wheat flour milling: A case study in particulate food processing. Pages 95-111 in: Powders and Solids-Developments in Handling and Processing Technologies. W. Hoyle, ed. RACI: London.

Creason, H. 1975. Grinding and corrugating rolls. Bull. Assoc. Oper. Millers November: 3569-3570.

Fang, C. Y., and Campbell, G. M. 2002. Stress-strain analysis and visual observation of wheat kernel breakage during roller milling using fluted rolls. Cereal Chem. 79:511-517.

Glenn, G. M., Younce, F. L., and Pitts, M. J. 1991. Fundamental physical properties characterizing the hardness of wheat endosperm. J. Cereal Sci. 13:179-194

Glenn, G. M., and Johnston, R. K. 1992. Moisture-dependent changes in the mechanical properties of isolated wheat bran. J. Cereal Sci. 15:223-236.

Haddad, Y., Mabille, F., Mermet, A., Abecassis, J., and Benet, J. C. 1999. Rheological properties of wheat endosperm with a view on grinding behaviour. Powder Technol. 105:89-94.

Martin, C., Rousser, R., and Brabec, D. L. 1993. Development of a single kernel wheat charactersation system. Trans. Am. Soc. Agric. Eng. 36:1399-1404.

[Received June 11, 2001. Accepted January 31, 2002.] 